**This is a draft version of a manuscript. Do not quote directly as content may change.**

The Psychological Science Collective: A collaborative data collection network to improve the generalizability of psychological science

Jonathon McPhetres $^{1 *}$ and Thuy-vy T. Nguyen ${ }^{1}$

${ }^{1}$ University of Rochester, Clinical and Social Sciences in Psychology, Rochester, NY, USA "Corresponding Author. Email: jon.mcphetres@gmail.com

Jonathon McPhetres is a PhD candidate at the University of Rochester in Rochester, NY, USA. Thuy-vy T. Nguyen is a Postdoctoral Researcher at the University of Rochester in Rochester, NY, USA.

Simulation file: https://osf.io/7462p/?view_only=0e5905f1a0f844ffad9c09cc266be2c9 


\begin{abstract}
In this manuscript, we describe a model for collaborative data collection. It is well known that reliance on small, homogeneous samples of western students severely undermines the generalizability and replicability of research in psychological science. Without widespread implementation of a system to improve how data is collected and accessed, researchers will continue to rely on convenience samples. Here, we propose the Psychological Science Collective (PSC) as a solution. The goal of the PSC is to raise the standards for data collection in the psychological sciences and make well-powered, representative samples more commonplace. Creating a self-sustaining network where collaborating labs can pool their resources and gain access to larger and more representative samples from around the world will result in a net increase in sample size and generalizability across the field as a whole. For example, simulations suggest that $\sim 150$ labs of different sizes can pool their resources to conduct around $\sim 50$ studies with an average sample size exceeding $N=500$ over the course of 12 weeks. This collaborative system is aimed at the "average" research project conducted by the "average" researcher. Thus, researchers at all career stages are encouraged to conduct research through the PSC rather than running the study in their own lab using convenience samples.
\end{abstract}




\section{The Psychological Science Collective: A collaborative data collection network to improve the generalizability of psychological science}

Clearly, one goal of psychological science is to understand humans in general (Rad, Martingano, \& Ginges, 2018). However, reliance on small convenience samples of western students severely undermines this goal and leads to larger problems of replicability. Though many researchers have begun discussing solutions to these problems, no clear solution has been implemented as a standard practice. One problem to consider is that any proposed changes to research standards (e.g. minimum sample sizes, requiring cross-cultural methods) essentially means that researchers with a lack of resources cannot contribute to psychological science if they cannot recruit large, multicultural samples (at least until egalitarian funding mechanisms becomes the norm: Vaesen \& Katzav, 2017). While suggestions have been made to improve psychological science as a whole, individual researchers still lack the ability to consistently implement many of these changes.

In the current paper, we reimagine the standard model of data collection in psychological science and propose a new network model: The Psychological Science Collective. In this model, laboratories from around the world can pool resources to collect data for each other in a selfsustaining, give-and-take model. The result is a worldwide network of labs-small, medium, and large - pooling resources and yielding large and representative samples in a relatively short period of time. This allows for more studies to be conducted by more research groups, and for each of those studies to be well-powered and representative. With this model, we hope to improve the way psychological science currently operates. Importantly, this network will be available for the average researcher and the average research study— that is, this network is not aimed only at large scale studies or registered reports, which may be intimidating for some 
researchers. Rather, with this model, we hope to change the way data is collected for most psychological studies.

Below, we first briefly outline what others have identified as shortcomings in the current model of psychological science and recent initiatives proposed to address those shortcomings. We then move on to discussing the Psych Science Collective model and how it addresses each of those shortcomings.

\section{Steps Towards Reforming Psychological Science}

The veracity and integrity of psychological science has come under scrutiny within the last decade. For example, a large portion of effects do not successfully replicate (Camerer et al., 2018; Klein et al., 2018; Open Science Collaboration, 2015), partially due to the combination of low statistical power, questionable research practices, and inappropriate generalization of results (for reviews of the 'crisis in social psychology', see Giner-Sorolla, 2019; Spellman, 2015). However, calls to address problems of low statistical power (Cohen, 1962; 1990; 1992), an overreliance on convenience samples (Ioannidis, 2005; Simons, Shoda \& Lindsay, 2017), and an over-reliance on Western, Educated, Industrialized, Rich and Democratic (WEIRD) samples (Henrich, Heine, \& Norenzayan, 2010a, 2010b) are not new (e.g., Greenwald, 1975; Sears, 1986; Sherif, 1970, 1977; Tversky \& Kahneman, 1971).

Many solutions have been proposed in response and some of these changes have been adopted. To deal with problems of low statistical power, for example, most journals now require a discussion of sample size and a priori power analyses or sensitivity analyses (eg, JESP editorial guidelines, 2016) and minimum sample sizes have been suggested (Simmons, Simonsohn, \& Nelson, 2011; Simmons, Nelson, \& Simonsohn, 2018). 
Responses to these solutions have run the gamut. On one end of the spectrum, it has been argued that psychological science should prioritize achieving a balance between Type 1 and Type 2 errors - that is, prioritizing exploration and net output over the integrity and replicability of published findings (Finkel, Eastwick, \& Reis, 2015, 2017). On the other end of the spectrum, researchers have argued for minimizing Type 1 errors, reasoning that reforming research practices does not banish exploratory research (LeBel, Campbell, \& Loving, 2017; LeBel, Berger, Campbell \& Loving, 2017). On both ends of this spectrum, however, scientists are realizing they need large and representative samples in order to answer the questions they want to answer. In other words, there can be no compromise: psychological research needs a solution which will lead to maximizing the cumulative generalizable body of knowledge while simultaneously maintaining the integrity of that body of knowledge.

Sample Size Solutions. Samples should be large enough to provide adequate power and representative enough to draw general conclusions about humans at large ( $\operatorname{Rad}$ et al., 2018). Psychologists have been critical of the reliance on samples of American undergraduate students since the 1970s (Sherif, 1977; Sears, 1986). Little has been done to address that concern as most published research relies either on student samples (Witte \& Brandt, 2011) or online samples (Andersen, Allen et al., 2018). Similarly, concerns about power have been voiced since the 60s (Cohen, 1962; Greenwald, 1987; Simmons et al., 2011, 2018) but very few changes have been consistently implemented as a result of these concerns.

One concern is that requiring large and representative samples increases the cost of studies beyond the resources available to most researchers. Though such changes may yield improvement in the long-term by increasing the quality of published research, it remains unclear how individual researchers can improve their practices - particularly those researchers with 
fewer resources and those from underdeveloped countries. Crowd-sourcing those large sample sizes seems to improve upon some of these limitations by combining the collective efforts of many laboratories around the world. However, researchers lack an accessible system which provides this sort of data collection on an ongoing basis.

The Rise of Collaborative Science and Mega Samples. The usual model of psychological science consists of an independent researcher or research group conducting their own studies with their own samples (usually local convenience samples). However, researchers have recently begun to discuss the benefits of crowdsourcing and collaboration (e.g., Uhlmann et al., 2018). Several organizations have recently formed which allow for "mega-samples" and large-scale replication efforts involving many laboratories around the world. For example, the Psych Science Accelerator (Moshontz et al., 2018) provides one solution to the above-mentioned problems by allowing for the collection of mega-samples within a relatively short time-frame. The Psych Science Accelerator organizes global data collection for specific large-scale studies which are democratically selected and are often accepted in-principal as registered reports. Study-Swap (https://osf.io/view/studyswap/) is another system allowing for researchers to collaborate on specific projects where one lab "needs" something that another lab "has." Study Swap puts individual researchers in contact with others who wish to collaborate and has previously allowed users to organize large-scale replication efforts. Another example is the Many Labs Collaboration (Open Science Collaboration, 2015; Klein et al, 2018), which focus exclusively on conducting replications of specific effects using hundreds of laboratories and often achieving sample sizes within the thousands.

However, none of these are self-sustaining systems for the "average" research project. The above three models cater towards large-scale studies which may be intimidating or not 
applicable to the average researcher. That's not to say the average researcher cannot spearhead a large-scale replication project, just that most researchers conduct smaller and more focused studies within their own research programs. If we are to improve psychological science as a whole, the average researcher needs the ability to obtain well-powered, heterogeneous (better yet, multi-national) samples for the average paper. Such a system needs to be accessible, affordable, and geared towards promoting the involvement of smaller researchers in underrepresented countries.

\section{The Psychological Science Collective}

Mission and Goals. The Psych Science Collective (PSC) is a network proposed to solve the above problems. As a currently hypothetical network, it lacks an organizational structure. However, as with any idea, a mission and a goal can be aimed towards in principle and can guide the development of the organization. The PSC is aimed at maximizing research output while achieving a balance between output and sample size so that every constituent can have access to large, representative samples. Thus, the mission of the PSC is as follows:

- To advance the current model of psychological science and to improve the generalizability and replicability of published research.

In service of this mission, the PSC has set out many goals to steer its decision-making processes. The goals of the PSC are as follows:

- To implement a fair and accessible data collection system which allows all researchers to obtain larger, representative samples.

- To address current shortcomings of psychological science including an over-reliance on underpowered and WEIRD convenience samples.

- To make large, multi-national samples the norm in psychological research. 
- To encourage participation and output from under-resourced labs.

- To encourage collaboration and resource-sharing across international borders.

- To discourage a culture of independent science which focuses on the accomplishments of individuals and to encourage a culture of collaborative science which focuses on the collective accomplishments of groups.

- To contribute to the normalization of open and transparent research and review practices.

\section{Overview of the PSC Model}

The PSC relies on a network of participating constituents of different sizes and capacities to collect data for each other in exchange for receiving data and/or authorship on projects in which they participate. Constituents will be sorted into three categories, small, medium, and large, based on the size of constituents' participation pools made available to the PSC by the principal investigator. The category into which constituents are sorted will indicate: 1) the likelihood of their proposals being accepted to receive data from the network, and 2) the length of time constituents will commit to collecting data for PSC.

The core idea of PSC network is that all constituents submitting proposals will enter with the knowledge that they will contribute data to the collective, regardless of whether their proposals pass the review process. Proposals which get selected will also commit to collecting data for other constituents in exchange for receiving data for their own projects. If a proposal is not accepted, a constituent participates in exchange for receiving authorship on the projects for which they collect data. Therefore, the model will consist of small, medium, or large constituents participating both as givers and takers. 
This "give-and-take" model will promote a fair distribution of benefits across constituents regardless of data collection capacities. More importantly, this system will make sure that medium and small constituents will benefit from being able to obtain larger sample sizes and more generalizable samples than what they would typically get from their participant pools. On the other hand, while large constituents might not increase their total available pool by participating, they will still benefit from receiving diverse and generalizable data collected by other constituents at a faster rate.

\section{Structure of the PSC Network}

The diagram below (Figure 1) represents a conceptual depiction of the PSC network. A given constituent, represented by the blue circle in the center, proposes a project to the PSC. When the project proposal gets accepted, other constituents will collect data for that project, either in exchange for receiving data of their own (the red labs) or in exchange for authorship on that project if their own proposal is not selected (the grey labs). In turn, the blue constituent will also collect data for selected projects from other labs, represented by the green circles, for a period of time. Likewise, the blue constituents will not be the only constituent collecting data for a given project, but rather the data will be crowdsourced from multiple constituents (not depicted) who are either participating in exchange for authorship or in exchange for receiving data.

This model is recursive: red constituents will be receiving data from other constituents and the green constituents will then be collecting data for other projects in exchange for receiving their own data. The amount of data a given constituent collects is determined by their available resources; data collection continues for a prespecified period of time. Thus, in a 12- 
week period, for example, a constituent may collect data for several projects and their

contribution may range from a few subjects per week to hundreds of subjects per week.

Fig. 1. Conceptual Diagram of the PSC. Network. Circles represent small, medium, and large-sized labs making up the PSC network; The blue circle represents a medium-sized lab which proposed a project to the PSC; the grey and red circles are labs collecting data for that project; some labs (grey) collect data in exchange for authorship and others (red) collect data in exchange for receiving data on their own projects. In exchange for receiving data from the network, the blue lab collects data for other projects proposed by the green circles.

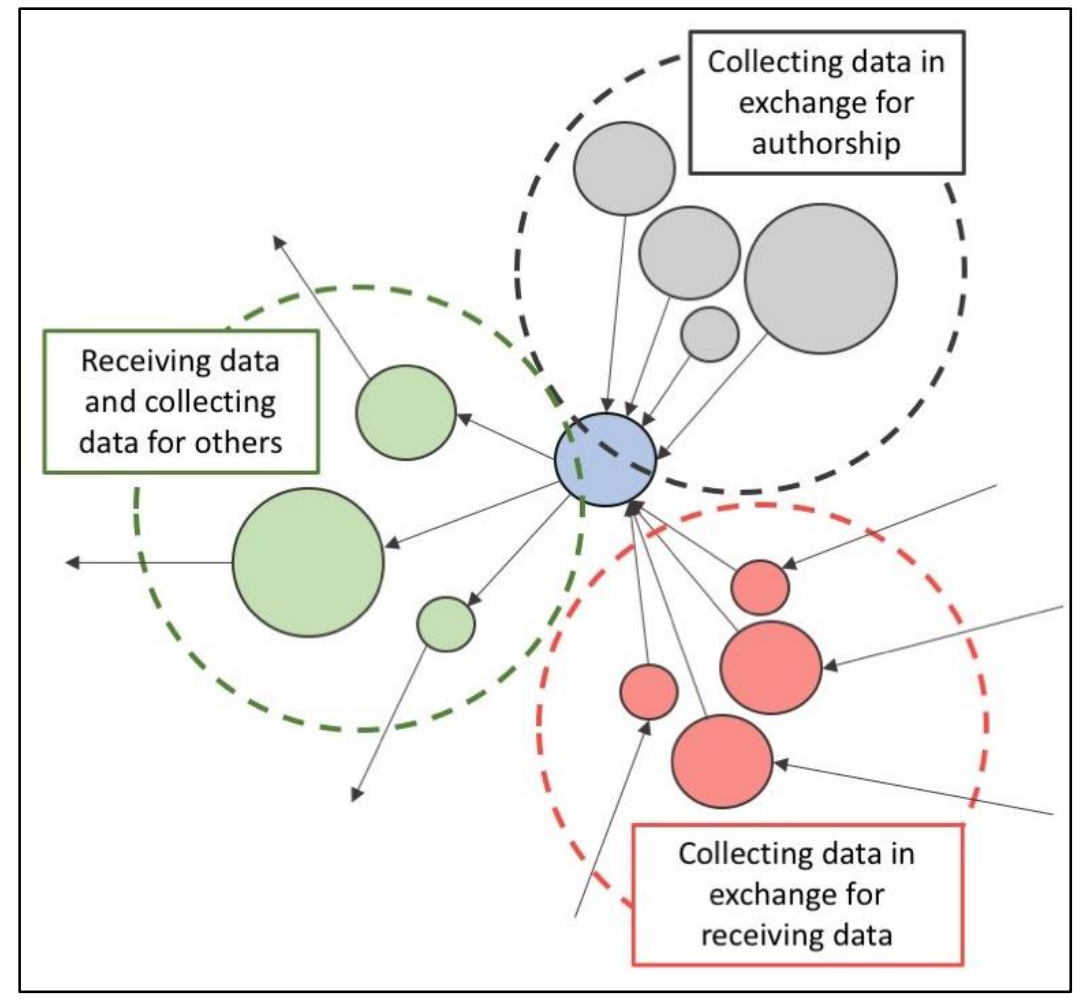

\section{Outline of Model Phases}

The PSC follows a four-phase process. These phases operate on a rolling basis staggered around a 12-week data collection period (an academic quarter). These phases are depicted in Figure 2, which follows the experiences of two hypothetical laboratories involved in the PSC. Each of the phases are explicated in the sections below. 
Fig 2. Depiction of the phases of the PSC process. The four phases of the PSC process operate on a rolling basis; the depiction illustrates the hypothetical experiences of three labs, one of which has a proposal accepted, one of which does not have a proposal accepted, and a third which does not submit a proposal.

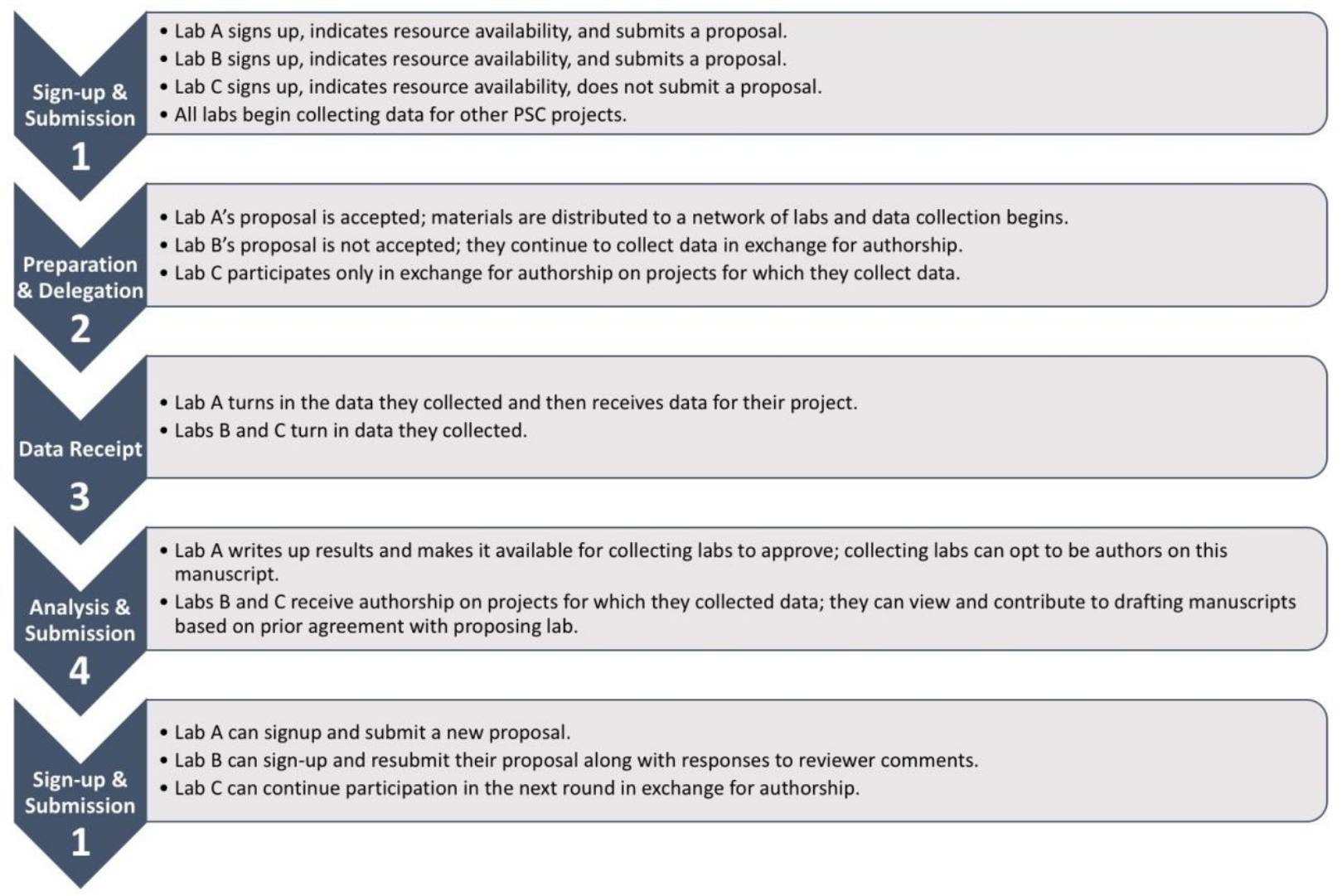

Phase 1: Sign-up and Submission. The first step in participating in the PSC network is

to submit a proposal for a study and to indicate the resources one has to offer. When submitting a proposal to be considered, the proposing constituent agrees to 1) to collect data for other constituents during that round of data collection regardless of whether their proposal is accepted, 2) review three other proposals, and 3) include as authors whichever constituents collect data for their project. Phase 1 has three stages: a feasibility assessment, a peer review stage, and an acceptance stage.

1. Feasibility assessment stage. The goal of this phase is to determine whether the proposal is "do-able" within the PSC network. A brief review of the methods, design, and requirement of the study will determine whether the proposal is suitable for the PSC. For 
example, proposals which require long periods of time, specialized equipment, or which lack certain elements of design or planning will not continue on to the review stage.

2. Peer review stage. Proposals will be distributed to participating constituents, and each proposal will receive at least three reviews. A standardized review process will be put in place and reviewers will score the proposals based on a few set criteria to minimize idiosyncrasy. This review process will be similar to that use in the stage 1 review of registered reports. For example, proposals will be evaluated on the proposed methodology, study design, and planned analysis and will not be evaluated based on importance, interest, or other subjective elements. The goal of the review stage is to provide a 1) constructive evaluation of the proposal, 2) an overall score used in determining acceptance, and 3) feedback for improving the project (if not accepted).

Reviewers are non-anonymous and proposal scores and reviewers' feedback to support their rating decisions will be made public if the proposal is accepted. However, reviewers will be blind to the proposing authors to minimize preference for specific authors, minimize bias for or against the questions being asked, and minimize preference for or against constituents with more or fewer resources or against any types of questions being asked.

3. Acceptance stage. The acceptance stage will be carried out by a committee of PSC members. Proposals will be ranked by the scores they receive during peer review, and only certain percentage of the highest-ranked proposals from each category (i.e., small, medium, or large) will be selected to receive data for that round. The threshold of scores will be predetermined based on the number of proposals submitted and the total available resources at a given time.

Acceptance rates and weights will be determined prior to submission and will be weighted such that larger constituents will have higher chance of being selected. In other words, 
as indicated in the Model Overview section above, acceptance rate will be partially determined by the number of subjects a specific constituent makes available to PSC network. This weighting can be adjusted according to the net subject pools available in order to maximize average sample size and number of studies outputted. In sum, the likelihood of proposal acceptance can be increased by the combinations of two factors: a strong, well-designed proposal and increasing the resources made available to the network.

Phase 2: Preparation and Delegation. The second step is for the study collection to be delegated to a network of constituents around the world. Regardless of whether or not a proposal is accepted, a proposing constituent will be asked to collect data for a specified period of time. The study materials and procedures will then need to be distributed to the participating constituents. All details of the project will need to be communicated by the proposing constituent - that is, the PSC serves to connect labs and moderate network activities, but will not be responsible for training, problem-solving, or other day-to-day responsibilities of carrying out the research.

Delegation of studies will be decided using an algorithm (to be designed) which considers three criteria: 1) the current number of submissions, 2) the available resources of participating labs, and 3) and an estimation of the time involvement and achievable sample size given criteria 1 and 2 .

Participating constituents will be required to collect data for a variable number of projects for a specified period of time. The period of involvement required can vary depending on the network availability at the time of proposal. However, the goal here is to achieve a balance between sample size and time requirements while maximizing research output. In simulations (https://osf.io/7462p/?view_only=0e5905f1a0f844ffad9c09cc266be2c9) we estimated that 
participation could require 12 weeks based on reasoning that a university quarter is 3 months and a semester is 4 months. Thus, the ability to achieve a large sample within this time frame is a benefit to any small or medium-sized constituents while the ability to achieve a multi-national sample in this timeframe is a benefit to a large-sized lab.

PSC will moderate material distribution relying on Open Science Framework as the main repository for all PSC projects. Materials, training protocols, and files shared between constituents for a specific project will be uploaded to a folder dedicated for that project on OSF. Materials and procedures may need to be translated and this effort will be the responsibility of the proposing lab, but such requirements may be crowd-sourced among the constituents that are involved in a specific project.

Phase 3: Data Receipt. At the end of the specified data collection period, data will be uploaded into a data repository where it will be distributed to the proposing laboratory once their obligations are fulfilled. At this point, the data may not be anonymized or de-identified, so it will not be publicly available. Instead, the PSC will maintain a confidential repository of all raw data on a private server. It will be the responsibility of the requesting laboratory to make the final, deidentified data publicly available (e.g., on the Open Science Framework or other public repository) within a specified time-frame.

Phase 4: Analysis and Submission. Upon receipt of data, constituents will then complete analysis of the data and will draft the final manuscript. Participation in this stage of the process has several requirements relevant to the other constituents involved in data collection.

First, all analysis code, materials, and data must be publicly available on a permanent repository and should follow the plans outlined in the initial submission as closely as possible. 
Any details of collaborative analyses are the responsibility of the leading authors and fall outside of the authority of the PSC, given that the following requirements are satisfied.

Second, leading authors are required to make their drafted manuscript available for viewing by those constituents who participate in data collection on a particular project. This can take the form of a private preprint, for example, or it can be a privately accessible document (e.g. Google doc). The goal here is to ensure that the output of the research is reported in a timely fashion and not file-drawered.

Third, all participating constituents must be offered authorship on projects for which they collect data (unless they also receive data for their own projects) and thus must be able to view and agree to the final manuscript before it is submitted. Of course, one is not required to accept authorship.

Fourth, once manuscripts are finalized, manuscripts must by default be available as publicly available preprints on an accepted server (e.g. OSF Preprints, PsyArxiv, etc) prior to journal submission. In the event an article is not to be submitted, the results must still be posted as a publicly available preprint. This will ensure that all results are reported and made publicly available regardless of the outcome and that participating constituents are recognized for their contributions.

Resubmission. If a proposal passes the feasibility stage but is not accepted during the acceptance stage, the proposal can be resubmitted again. The authors will be encouraged to incorporate the feedback from the reviewers to increase their scores during the second round of reviews. To encourage continued participation, resubmitted proposals will be considered along with comments to the first round of reviews and will be considered as a "revise and resubmit."

\section{Model Benefits and Incentives}


Given the mission and goals outlined above, the PSC model has several aims. The first is to create and maintain a self-sustaining system which can become the standard for how data is collected. The second is to achieve a balance between net research output and sample size while minimizing the time required to achieve that data. The third is to maximize involvement; the PSC is designed so that all labs, regardless of size and resources, will benefit in some way. This is a lot to juggle, but the benefit for individual constituents far outweigh the cost. As such, we outline here the benefits and incentives implicit in this model.

Give-and-take model. The PSC is based on an egalitarian, give-and-take model where one must give some data in order to receive data. Ultimately, this model relies on the idea that one would be likely be collecting data anyway, but that in a period of time where a small constituent may only be able to collect data from 100 subjects, for example, they will now receive data from several hundred subjects. Similarly, in a period of time where a large constituent may be able to collect data from several hundred subjects, they now are able to receive data from several hundred subjects around the world.

Increasing the average sample size. The goal of the PSC is to achieve a balance between maximizing research output and sample size. A power analysis using G*Power (Faul, Erdfelder, Buchner \& Lang, 2009) indicates that to detect a small difference between two conditions $(d=.20)$ with $80 \%$ using a one-tailed $t$-test, a sample size of 620 is needed. As a minimum standard, the goal is to achieve sample-sizes of at least $N=500$ for each study which would nearly triple the suggested minimum cell size of 60 (Simons et al., 2018) in a study with three conditions, for example. It would then be considered an achievement if every study ever published had at least this minimum sample size. 
To demonstrate that the PSC model will generate sample sizes around 500 per study, we conducted a series of simulations (accessible on the Open Science Framework at the following link: https://osf.io/7462p/?view only=0e5905f1a0f844ffad9c09cc266be2c9) and manipulated the following variables:

- Numbers of small, medium, and large constituents in the entire network (cells $\mathrm{B}_{4}, \mathrm{~B}_{5}, \mathrm{~B}_{6}$, respectively); this will be the total from which a random number from each category will be drawn to be set as the number of constituents from each category that submit proposals during one round of submission.

- Acceptance rates for each category for submissions (cells $\mathrm{C}_{4}, \mathrm{C}_{5}, \mathrm{C}_{6}$, respectively).

- The average sample sizes that small, medium, and large constituents (cells $\mathrm{D}_{4}, \mathrm{C}_{5}, \mathrm{C}_{6}$, respectively) can contribute per 12 weeks (i.e., academic quarter)

- The length of time that small, medium, and large constituents will commit to collecting data for PSC projects (cells $\mathrm{E}_{4}, \mathrm{E}_{5}, \mathrm{E}_{6}$, respectively)

Simulations can be run to see which combinations of these variables will yield average sample sizes around $N=500$.

Removing researcher bias, effect allegiance, and optional stopping. Other models of collaborative science allow researchers to opt-in to collect data for specific projects which they are interested in. While the is the usual model of psychological science (i.e. one studies what they are interested in) it is well known that researcher bias can influence the effects obtained. Further, meta-analyses often find that researcher bias, demand characteristics, and effect allegiance — whether one believes an effect works or not — explains some variance across studies (for an example in clinical sciences, see Lubborsky et al., 1999). The proposed PSC model would pseudo-randomly assigns studies to labs. A researcher's time, therefore, is devoted to 
collecting data as they normally would - just on projects which they are likely not invested in the outcome. Further, this model eliminates the possibility of optional stopping based on preliminary analyses because the data will be collected by other researchers.

Promoting transparent science. By incorporating a pre-review process and requiring transparent practices, the PSC normalizes many of the recent changes implemented into the field of psychology. The PSC will utilize the Open Science Framework as the main repository for sharing materials, data, code, and preprints.

Providing authorship, training, and experience. There are many reasons a given constituent may want to participate in the PSC. Some constituents may also simply want to participate in exchange for authorship on a paper. Likewise, first-year graduate students, undergraduate research methods or experimental psychology classes, honor students, or thesis groups may wish to participate as a learning experience. Participating in collaborative science not only provides hands-on research training for students, but also provides experience managing data, communicating with collaborators, and provides an opportunity for students to learn about the publication process.

Benefits for small and medium-sized labs. Smaller constituents often lack the resources to run even small studies. A collaborative network allows for under-resourced constituents to participate in psychological science. As a metaphor, philosophers often wonder how many Einsteins and Mozarts are born poor and therefore not able to discover or compose symphonies. In this same way, we scientists must wonder how many great ideas are abandoned because of a lack of resources. This network therefore benefits small constituents directly, but ultimately benefits science as a whole by including those who were previously absent from science. 
Benefits and expectations for large labs. Large constituents will likely be able to pursue multiple projects at once. As such, larger constituents can contribute more subjects in less time and, given a simple study design, will be a necessary contributor to many proposed projects. That is, the PSC may provide an opportunity for a larger constituent to conduct a cross-cultural study while also pursuing additional projects. Put differently, in the same period of time where a constituent may be able to collect data from several hundred homogeneous undergraduates, they can now receive data from several hundred subjects from around the world.

\section{Addressing Possible Concerns}

Psychology is a relatively young field in comparison to other sciences. As such, we are simultaneously dealing with some old problems and some very new problems — we have to learn how to do science our way. During this process, some concerns and challenges may arise. Many of these challenges are being tackled by current and ongoing collaborations. For example, the logistics of running studies across international borders, managing ethics approvals, translating materials into multiple languages, incorporating under-resourced labs, and managing data are issues faced by many collaborative studies. The PSC could provide a way of streamlining such issues. Further, the PSC could also provide a systematic way of streamlining the dissemination of materials and data and opening the door for training researchers from under-resourced regions.

The largest challenge the PSC model will face is creating the infrastructure. Much of what a network like the PSC relies on is currently available through systems like the Open Science Framework. However, a team of leaders will be needed to guide the decision-making process and other individuals will be needed to oversee submission and delegation of studies, and communications between participating labs. 
Corollary to this challenge is increasing the participation of labs with no access to free participant samples. Funding (to pay participants) may be required in the future to increase the likelihood that labs from non-western countries will participate. Further, while the current design of the PSC imagines that most research will be social-psychological in nature, labs wishing to conduct more intensive studies will need funding to provide equipment and training to other labs collecting data.

Other challenges concern elements of the PSC design. For example, how much will a non-anonymous review process persuade or dissuade participation? How much work are researchers willing to give in exchange for receiving data? How likely are constituents to resubmit an unsuccessful proposal? Additionally, in an effort to create a system that operates on a continuous basis we must maximize the number of projects completed while also maintaining large sample sizes for everyone. At the same time, we must prioritize encouraging participation from smaller constituents while also being cognizant of the fact that larger constituents contribute the most. Compromises must be made to achieve all of these at once; we think that minimum sample sizes and maximum time frames are a good standard to aspire to and will provide a workable framework. The mission statement and goals are likely good guides when considering these challenges.

Another challenge is when new proposals are accepted and the timeline for beginning each round of data collection. One solution is to carry out three rounds per year and to stagger each round. This would allow data collection to be carried out during the normal fall and spring academic semesters/quarters, plus an additional round during the summer. However, a summer round would likely be slow and may garner lower participation rates. Ultimately, the most productive time periods will need to be identified through practice. 
A final challenge is 'ramping up' participation so that the desired sample sizes and participating constituents are consistently available. It is anticipated that such a model would be of interest to many labs, large and small. However, as with any new innovation it will likely take time to achieve maximum participation. Thus, one challenge will be the logistics of planning and delegating data collection among an ever-changing pool of participating constituents.

\section{What kinds of proposals are suitable for the PSC?}

Other collaborative networks (e.g., Study Swap, Many Labs, and Psych Science Accelerator) geared towards large-scale registered reports and high-profile studies, which may be intimating or not applicable to mot researchers. However, the PSC is geared towards the average, everyday project. The goal of the PSC is to increase the quality of all science, not to increase the frequency of a specific type of science. Thus, researchers are encouraged to submit their "everyday" projects to the PSC rather than running them on their own or using convenience samples.

Who should submit proposals to the PSC? All researchers are encouraged to submit proposals to the PSC, regardless of rank, affiliation, or resources. Further, pilot data and registered report acceptance would not required for a proposal, although elements such as preregistration and open materials and data would be inherently necessary.

Who can participate in the PSC? Any researcher with the ability to contribute to data collection can participate. A proposal is not required to participate in the PSC; one could participate with the intention of receiving authorship in exchange for collecting data. Such incentives are already observed in large-scale replication rings. Further, such participation may benefit undergraduate research methods courses and can provide a hands-on learning experience to grad students or those in smaller departments who contribute less actively to research. 


\section{Final Comments}

The mission of the PSC is to improve the way psychological science is conducted and to create a new standard for how data is collected. A science of humans cannot draw conclusions from convenience samples collected by one individual lab in one region. A science of humans needs to aim towards robust samples and generalizable conclusions. Further, the ability to draw generalizable conclusions should be a characteristic of all published work and thus should be attainable by all scientists, not only a privileged few.

Science is no longer an independent activity - it no longer takes place behind closed doors and no longer rewards a single individual for the efforts of many. Science is collaborative and relies on the efforts of many, so all should benefit. The PSC model addresses many of the concerns, shortcomings, and problems facing psychological science today. While it does not solve every problem, it is a much-needed step in changing the way we do science.

\section{References}

Anderson, C. A., Allen, J. J., Plante, C., Quigley-McBride, A., Lovett, A., \& Rokkum, J. N. (2018). The MTurkification of social and personality psychology. Personality and Social Psychology Bulletin, online publication. Retrieved from http://journals.sagepub.com /doi/abs/10.1177/0146167218798821.

Camerer, C. F., Dreber, A., Holzmeister, F., Ho, T. H., Huber, J., Johannesson, M., ... \& Altmejd, A. (2018). Evaluating the replicability of social science experiments in Nature and Science between 2010 and 2015. Nature Human Behaviour, 2(9), 637.

Cohen, J. (1962). The statistical power of abnormal-social psychological research: a review. The Journal of Abnormal and Social Psychology, 65(3), 145. 
Cohen, J. (1990). Things I have learned (so far). American psychologist, 45(12), 1304.

Cohen, J. (1992). A power primer. Psychological bulletin, 112(1), 155.

Faul, F., Erdfelder, E., Buchner, A., \& Lang, A. G. (2009). Statistical power analyses using G* Power 3.1: Tests for correlation and regression analyses. Behavior research methods, 41(4), 1149-1160.

Finkel, E. J., Eastwick, P. W., \& Reis, H. T. (2015). Best research practices in psychology: Illustrating epistemological and pragmatic considerations with the case of relationship science. Journal of personality and social psychology, 108(2), 275.

Finkel, E. J., Eastwick, P. W., \& Reis, H. T. (2017). Replicability and other features of a highquality science: Toward a balanced and empirical approach. Journal of Personality and Social Psychology, 113(2), 244.

Giner-Sorolla, R. (2019). From crisis of evidence to a "crisis" of relevance? Incentive-based answers for social psychology's perennial relevance worries. European Review Of Social Psychology, 30, 1-38. doi:10.1080/10463283.2018.1542902

Greenwald, A. G. (1975). Consequences of prejudice against the null hypothesis. Psychological bulletin, 82(1), 1.

Henrich, J., Heine, S. J., \& Norenzayan, A. (2010a). The weirdest people in the world?. Behavioral and brain sciences, 33(2-3), 61-83.

Henrich, J., Heine, S. J., \& Norenzayan, A. (2010b). Most people are not WEIRD. Nature, 466(7302), 29.

Ioannidis, J. P. (2005). Why most published research findings are false. PLoS medicine, 2(8), e124. 
Klein, R. A. (2018). Many Labs 2: Investigating variation in replicability across sample and setting.

LeBel, E. P., Berger, D., Campbell, L., \& Loving, T. J. (2017). Falsifiability is not optional. Journal of Peronality and Social Psychology, 113, 254-261.

LeBel, E. P., Campbell, L., \& Loving, T. J. (2017). Benefits of open and high-powered research outweigh costs. Journal of personality and social psychology, 113(2), 230.

Luborsky, L., Diguer, L., Seligman, D. A., Rosenthal, R., Krause, E. D., Johnson, S., ... \& Schweizer, E. (1999). The researcher's own therapy allegiances: A "wild card" in comparisons of treatment efficacy. Clinical Psychology: Science and Practice, 6(1), 95106.

Moshontz, H., Campbell, L., Ebersole, C. R., IJzerman, H., Urry, H. L., Forscher, P. S., ... \& Castille, C. M. (2018). The Psychological Science Accelerator: Advancing psychology through a distributed collaborative network. Advances in Methods and Practices in Psychological Science, 2515245918797607.

Open Science Collaboration. (2015). Estimating the reproducibility of psychological science. Science, 349(6251), aac4716.

Rad, M. S., Martingano, A. J., \& Ginges, J. (2018). Toward a psychology of Homo sapiens: Making psychological science more representative of the human population. Proceedings of the National Academy of Sciences, 115(45), 11401-11405.

Sears, D. O. (1986). College sophomores in the laboratory: Influences of a narrow data base on social psychology's view of human nature. Journal of Personality and Social Psychology, 51, 515-530. 
Sherif, M. (1970). On the relevance of social psychology. American Psychologist, 25, 144-156. http://doi.org/10.1037/h0029429

Sherif, M. (1977). Crisis in social psychology: Some remarks towards breaking through the crisis. Personality and Social Psychology Bulletin, 3, 368-382.

Simmons, J. P., Nelson, L. D., \& Simonsohn, U. (2011). False-positive psychology: Undisclosed flexibility in data collection and analysis allows presenting anything as significant. Psychological science, 22(11), 1359-1366.

Simmons, J. P., Nelson, L. D., \& Simonsohn, U. (2018). False-positive citations. Perspectives on Psychological Science, 13(2), 255-259.

Simons, D. J., Shoda, Y., \& Lindsay, D. S. (2017). Constraints on generality (COG): A proposed addition to all empirical papers. Perspectives on Psychological Science, 12(6), 11231128.

Spellman, B. A. (2015). A short (personal) future history of revolution 2.0. Perspectives on Psychological Science, 10, 886-899.

Tversky, A., \& Kahneman, D. (1971). Belief in the law of small numbers. Psychological bulletin, 76(2), 105.

Uhlmann, E. L., Chartier, C. R., Ebersole, C. R., Errington, T. M., Kidwell, M., Lai, C. K., ... Nosek, B. A. (2018, August 13). Scientific Utopia: III. Crowdsourcing Science. https://doi.org/10.31234/osf.io/vg649

Vaesen, K., \& Katzav, J. (2017). How much would each researcher receive if competitive government research funding were distributed equally among researchers?. PloS one, 12(9), e0183967. 
Witte, E. H., \& Brandt, V. (2011). Social psychological research: The comparison of four journals. Retrieved from http://psydok.sulb.uni-saarland.de/volltexte/2011/2743 